\title{
Interference and Resource Management Through Sleep Mode Selection in Heterogeneous Networks
}

DOI:

10.1109/TCOMM.2016.2623614

\section{Document Version}

Accepted author manuscript

Link to publication record in Manchester Research Explorer

\section{Citation for published version (APA):}

Ebrahim, A., \& Alsusa, E. (2017). Interference and Resource Management Through Sleep Mode Selection in Heterogeneous Networks. IEEE Transactions on Communications, 65(1), 257 - 269.

https://doi.org/10.1109/TCOMM.2016.2623614

\section{Published in:}

IEEE Transactions on Communications

\section{Citing this paper}

Please note that where the full-text provided on Manchester Research Explorer is the Author Accepted Manuscript or Proof version this may differ from the final Published version. If citing, it is advised that you check and use the publisher's definitive version.

\section{General rights}

Copyright and moral rights for the publications made accessible in the Research Explorer are retained by the authors and/or other copyright owners and it is a condition of accessing publications that users recognise and abide by the legal requirements associated with these rights.

\section{Takedown policy}

If you believe that this document breaches copyright please refer to the University of Manchester's Takedown Procedures [http://man.ac.uk/04Y6Bo] or contact uml.scholarlycommunications@manchester.ac.uk providing relevant details, so we can investigate your claim.

\section{OPEN ACCESS}




\title{
Interference and Resource Management Through Sleep Mode Selection in Heterogeneous Networks
}

\author{
Aysha Ebrahim, Student Member, IEEE, and Emad Alsusa, Senior Member, IEEE
}

\begin{abstract}
This paper investigates the capacity and energy consumption metrics of small-cell networks that are enabled with sleep mode (SL) functionality. A novel method is introduced to systematically and accurately identify the potential SL cells that can maximize the spectrum reuse efficiency without the need for an exhaustive search. The performance of the proposed technique is assessed and compared with the always-on approach and an optimal benchmark. The results show that the proposed method significantly outperforms the always-on system and approaches the performance of the optimal benchmark with notably reduced computational burden.
\end{abstract}

Index Terms-Femtocell, Heterogeneous Network, Interference Avoidance, LTE, OFDMA, Resource Management, Sleep Mode

\section{INTRODUCTION}

The vast requirement for wireless data and voice has increased the pressure on the radio access links and backhaul infrastructure of cellular networks. This poses a major challenge for the operators who serve a growing number of subscribers. While Long-Term Evolution (LTE) systems can provide higher data rates than $3 \mathrm{G}$, the expected future capacity requirements cannot be satisfied even though LTE enjoys a wider bandwidth. Several solutions were introduced to enhance the capacity of such networks including adding more macrocells and cell splitting. However, those solutions are not cost effective and require high Capital Costs (CAPEX) and Operational Costs (OPEX). An alternative approach is to apply cell-densification within heterogeneous networks, where the number of low-cost cells, such as pico- and femto-cells, deployed within the coverage area of macrocell networks is increased to enhance capacity and coverage [1].

However, the unplanned distribution of small cells poses many threats for the network operators. In particular, cross-tier and co-tier interference is a major challenge that results from the unplanned deployment [2]. Co-tier interference results from the densification of small cells which generates a large number of overlapped cell boundaries. On the other hand, excessive cross-tier interference caused by the macrocell can seriously degrade the performance of the low power cells. To overcome such issues, network operators must utilize efficient radio resource management (RRM) techniques. Intercell interference coordination is one form of RRM that is used to control interference by allocating users orthogonally in the time and frequency domains such that potential interference is prevented [3]. As interference may degrade the overall performance of heterogeneous networks, special care should be given to cross-tier interference mitigation. Fractional frequency reuse (FFR) is one of the popular inter-cell interference coordination (ICIC) techniques in heterogeneous networks due to its low complexity and limited cooperation required between BSs [4].

To achieve further performance gains, new strategies need to be utilized in combination with RRM techniques. Sleep mode is a promising solution for improving energy efficiency and capacity in cellular networks as studies show that around 10$60 \%$ energy saving can be achieved with such techniques [5]. A small cell can be set to operate in ready mode (RE) or sleep mode (SL) [5]. In RE, all the hardware parts of a small cell are switched on whilst in SL, part of the hardware components can operate in low power while other parts are fully switched off. The decision of which parts to be switched off is determined by the energy saving algorithm [5]. The transition between SL and RE states can be controlled either by the small cell, the user, or the core network. In small cell controlled SL mode, the small cell can turn off the pilot transmission when it senses no active mobile users using a low-power sniffing capability. In core network controlled SL, the transition between states is carried out by the core network via the back-haul. In the case of the user controlled SL a user can wake-up nearby small cells by broadcasting wake-up signals.

Recently, SL has been proposed for optimizing various aspects in cellular networks. In [6], the authors provided a survey on energy efficient techniques and power consumption models for enhancing the energy efficiency in femtocell networks. The authors in [7] presented a method for minimizing energy in heterogeneous networks by proposing a joint resource partitioning and user association method under the assumption that both tiers of cells can be switched into SL for a certain fraction of time/frequency. In [8], an SL based mechanism for minimizing energy consumption and maintaining QoS is proposed for heterogeneous networks. In this technique, macrocells are put into SL and users are offloaded to neighboring macrocells or small cells. The authors in [9] proposed a method for minimizing total power consumption in heterogeneous networks by adaptively switching BSs on/off taking into consideration parameters such as network coverage and the user traffic rates. The work in [10] introduced an SL method for studying the impact of switching off BSs on the capacity and energy efficiency in a homogeneous network of femtocells. An SL based technique was investigated in [11] to assess the energy efficiency and the capacity of the system. Different elements were taken into consideration to test the QoS including the transmission power of femtocells, the separation between BSs and the total quantity of femtocells. The authors in [12] presented a collaborative SL scheme that focuses on enhancing the energy saving of femtocell assisted networks by only activating the necessary femtocells 
to serve the users requirements. In [13], the authors introduced a cell selection scheme for scaling down the number of active femtocells while considering the QoS requirements of users. A heuristic algorithm is presented in [14] to reduce the power consumption by setting as many BSs as possible to SL to serve the users rate requirements. To ensure the minimum number of cells are active, the BSs offering acceptable service to the largest number of users are activated first. In [15], the authors proposed a SL activation method for two-tier cellular networks to reduce the interference to macrocell users.

The majority of the techniques that employ SL strategies aim to minimize energy consumption by exploiting the idle times, when the small cells have no active users, and setting them to SL mode to save power. In addition to minimizing power consumption, this paper proposes a novel SL-based RRM to leverage the capacity gain of cellular heterogeneous networks employing FFR. The motivation therefore is to exploit the ad-hoc nature of femtocell deployment and the limited percentage of associated users, to examine the possibility of temporary cell switch off as an additional degree of freedom in the optimization of the available resources. This is achieved by reducing the interference that is produced by poorly positioned small cells to allow more effective resource utilization in that part of the network. A resource partitioning scheme is incorporated in our work to control the inter-cell interference by orthogonalizing the interfering cells/users. To compensate for the reuse reduction that results from orthogonalization, we introduce a small cell selection algorithm which uses a set of criteria to identify the BSs that are causing the most reuse reduction and feeds this information to the resource partitioning scheme to determine whether or not if deactivation can improve the network resource utilization. It is shown that the proposed method improves the overall throughput as well as the energy efficiency performance of the system at low complexity and allows fast deactivation decisions using limited information exchanged between the central unit and the BSs. The performance of the proposed method is analyzed using a mathematical model which reveals an interesting insight into the superior performance of such systems and is verified by a good match between the analytical and simulation results. The main contributions in this work are:

- Formulation of a novel criterion for identifying interference scenarios in which switching off certain small cells can enhance the network capacity.

- Devised an effective SL-mode based resource allocation scheme with enhanced resource re-utilization gain.

- Presented a mathematical model that accurately captures the received interference and derived the upper bound performance that verify the anticipated gains.

The remaining part of the paper is structured according to the following. Section II provides a description of the system model and assumptions. Section III and IV illustrates detailed discussion of the proposed method. Section V presents a performance analysis of our proposed method. The simulation and result discussion are discussed in section VI. Lastly; concluding remarks are presented in section VII.

\section{SYSTEM MODEL AND ASSUMPTIONS}

A Downlink OFDMA system is utilized with a heterogeneous network with a macrocell and a group of small cells. Assume $\mathcal{B}$ and $\mathcal{K}$ denote the sets of deployed small cells and UEs in the network in which $\mathcal{B}=\{1,2, \ldots, i, \ldots, j, \ldots, B\}$ and $\mathcal{K}=\{1,2, \ldots, k, \ldots, K\}$. Let $i \in \mathcal{B}$ and $k \in \mathcal{K}$ represent any random BS and UE from the sets $\mathcal{B}$ and $\mathcal{K}$ and $j$ denote a neighboring cell of $\mathrm{BS} i$, where a neighboring $\mathrm{BS}$ is a nearby cell in which a UE $k$ served by BS $i$ falls within its coverage range according to a predefined threshold. Furthermore, let $\mathcal{S}_{i}$ refer to the group of UEs served by base station $i$. The system bandwidth is split into $N$ physical resource blocks (PRB) which are assumed to be orthogonal and represented by $\mathcal{N}=\{1,2, \ldots, n, \ldots, N\}$. Both the macrocell and small cells utilize a reuse- 1 system where all tiers of cells coexist in the same frequency band. Small cells are assumed to use an open access policy and are transmitting at a fixed power level. Moreover, users are associated with cells based on the maximum reference signal received power (RSRP) strategy [16]. The macrocell and picocells are connected to the core network through the radio network controller (RNC) and the femtocells are linked with the femtocell gateway through the internet. The Macro- and Pico- cells are managed by the operation and management unit (OAM) and the femtocell gateway is responsible for connecting the femtocells to the femtocell management system (FMS) which also uses the OAM unit to manage all the femtocells in the system. Therefore, the centralized control required for all BSs in the heterogeneous network is provided by both the OMS and FMS [17] [18]. Small cells can operate either in RE state or SL state where the transition between states is controlled by the core network using a wake-up control message via the backhaul [5]. Additionally, users send Channel Quality Indicator (CQI) reports to their serving BSs to indicate the quality of the communication channel. The signal-to-interference-plusnoise ratio (SINR) of user $k$ at $\operatorname{PRB} n$ is determined by [19]

$$
\Upsilon_{k, n}=\frac{\rho_{i, k, n} \cdot \hbar_{i, k, n}}{\eta_{0}+\sum_{j=1}^{B} \rho_{j, k, n} \cdot \hbar_{j, k, n}}, \quad j \neq i
$$

where $\rho_{i, k, n}$ and $\hbar_{i, k, n}$ denote the received signal power and the channel gain from BS $i$ to the served user $k$ on PRB $n$ respectively. $\rho_{j, k, n}$ is the interference power received from a neighboring cell $j$ and $\eta_{0}$ refers to the AWGN noise.

\section{INTERFERENCE MAP APPROACH}

To identify possible conflicts in the system, the interference map approach in [19] is employed. To build the interference map, BSs use measurement reports (MRs) acquired from their UEs and transmit radio resource control (RRC) messages to the UEs to trigger the MRs. UEs then scan the neighborhood to determine the physical cell identity (PCI) of neighboring BSs as well as their corresponding RSRP, which are reported back to their serving BSs [16]. Given this information, the local interference map, $\zeta_{i}$, , of BS $i$ is produced as follows [19] 


$$
\zeta_{i}(k, j)=\left\{\begin{array}{ll}
0 & \frac{\Gamma_{i, k}}{\Gamma_{j, k}}>\beta \\
1 & \frac{\Gamma_{i, k}}{\Gamma_{j, k}}<\beta
\end{array},\right.
$$

where $k$ denotes a user served by BS $i$ and $j$ is a neighboring BS of $i$. $\beta$ refers to the signal-to-interference (SIR) threshold, $\Gamma_{i, k}$ denotes the received power from BS $i$ to its connected $\mathrm{UE} k, \Gamma_{j, k}$ represents the interference power received by UE $k$ from BS $j$. The value of the threshold can be selected depending on the required optimization objective. Generally, higher threshold values are used for maximizing the QoS while lower values are used for maximizing the throughput. For further discussion on this please refer to sec.V of [19].

When a BS updates its local interference map, the current measurement, $\zeta_{i}$, is sent to the FMS only if the previous record, $\zeta_{i}^{\prime}$, is different from the current record [19]

$$
\sum_{k=1}^{\mathcal{S}_{i}} \sum_{i=1}^{B}\left|\zeta_{i}(k, i)-\zeta_{i}^{\prime}(k, i)\right|>0,
$$

The universal interference map of the network is managed by the FMS, where $\zeta \in \mathbb{R}^{K \times B}$ is expressed as [19]

$$
\begin{array}{lc}
\zeta(k, i)=w, & \text { when i is the serving BS of } k \\
\zeta(k, j)=1, & \text { when } j \text { interferes } k \\
\zeta(k, j)=0, & \text { otherwise }
\end{array} .
$$

where $w$ represent any positive integer number excluding 0 and 1 .

\section{The Proposed Method}

The main concept of the proposed scheme is to identify the scenarios in which deactivating certain small cells can be beneficial from a network-wide perspective. Towards this end, an algorithm is proposed to nominate the potential BSs, (e.g., BS $f_{1}$ from fig. 1), to the centralized resource allocation scheme to determine if deactivating these cells can result in improving the network-wide frequency reuse and hence capacity. If some cells are selected for deactivation, the resource allocation scheme will re-associate their users to neighboring cells and resolve any remaining co-tier interference instances through orthogonal resource partitioning. Due to the unplanned deployment of femtocells, the FMS is used in this algorithm as a centralized controller.

\section{A. SL for Capacity Maximization}

Our objective is to find the set of BSs which, if deactivated, could maximize the total capacity of the network. Therefore, the capacity maximization problem is formulated in this section. Generally speaking, we presume a BS operates either in active or sleep mode and the state of a BS is denoted by the indicator variable $\phi_{i} \in\{0,1\}$, where $\phi_{i}=1$ indicates active state and $\phi_{i}=0$ indicates sleep state. The achievable capacity, $c_{k, n}$ of $\mathrm{UE} k$ at PRB $n$ is given by

$$
c_{k, n}=W \cdot \log \left(1+\Upsilon_{k, n}\right)
$$

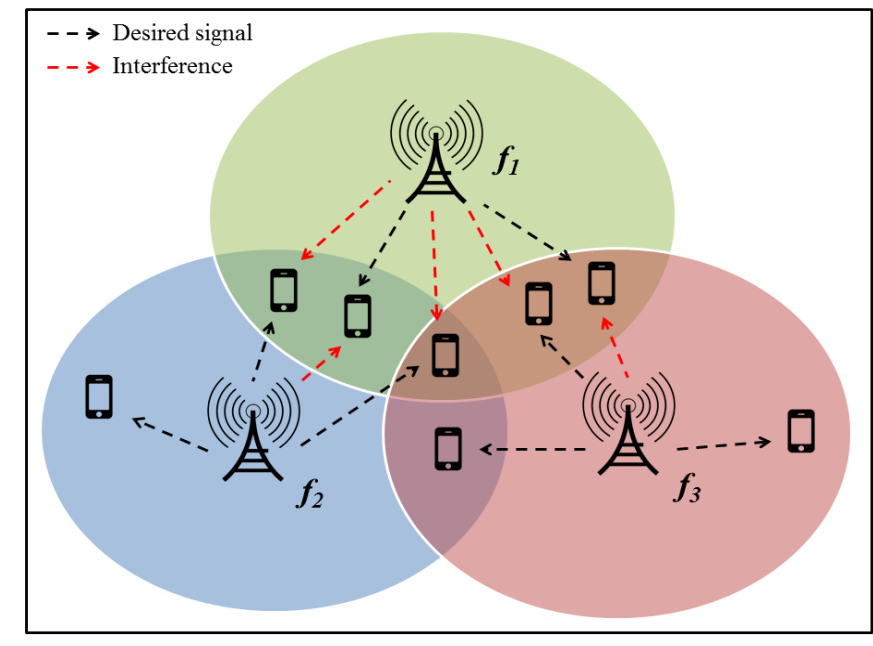

Figure 1. Illustration of cell switch-off scenario

where $W$ refers to the bandwidth of PRB $n$ which is identical for all PRBs. The capacity maximization problem can be formulated as follows

$$
\begin{array}{ll}
\max _{\phi, \chi} & \sum_{i=1}^{B} \sum_{k=1}^{K} \sum_{n=1}^{N} \phi_{i} \cdot c_{k, n} \cdot \chi_{k, n} \\
\text { s.t. } & \sum_{k=1}^{K} \chi_{k, n} \leq 1, \quad \forall i, n \\
& \sum_{n=1}^{N} \chi_{k, n}=\Psi_{k}, \forall k .
\end{array}
$$

where $\chi_{k, n}$ is an indicator variable that is equal to 1 when UE $k$ is allocated in PRB $n$ and 0 otherwise. The notation $\Psi_{k}$ refers to the amount of resources assigned to user $k$ which is determined based on sec. IV-C. (7) ensures that each PRB is allocated to one UE only in the same cell. (8) guarantees that UE $k$ is allocated $\Psi_{k}$ PRBs. The problem in (6) is formulated as an integer linear programming (ILP) problem which is normally solved by ILP solving methods. Since the running time of ILP solvers is uncertain, more efficient techniques are needed to reduce the complexity. We propose a unique solution that divides the problem into two parts. The first part is solved by the FMS which uses a set of criteria, to be defined later, to determine the potential BSs to be deactivated. This is assisted by a resource partitioning scheme that is used to determine whether or not switching off the selected BS's can lead to increasing the resource utilization. The second part is the resource allocation within each BS, which is solved independently by each BS using the CQI reports from users and the output of the first part including the set of active BSs and the advised allocated PRBs per user. It is worth highlighting that resource partitioning involves determining the amount of resources to allocate to each user to ensure orthogonality while the resource allocation is performed by the BSs to allocate their users in the preferred resources according to the wireless channel information. 


\section{B. Small Cell Deactivation Criteria}

This section defines the small cell deactivation criteria that will be used in sec. IV-D to select the candidate BSs for switch off. The key deactivation tests can be listed as follows:

Test 1: The number of conflicts (NoC) of a BS, which is given by the total amount of interfered UEs by BS $i$, in addition to the number of $i$ 's connected UEs that are interfered by nearby BSs. The FMS measures the NoC associated with each BS based on the interference map $\zeta$, as follows

$$
\operatorname{NoC}_{i}=\sum_{k=1}^{K}\{\zeta(k, i)=1\}+\sum_{\substack{j=1 \\ j \neq i}}^{B} \sum_{k=1}^{\mathcal{S}_{i}}\{\zeta(k, j)=1\}
$$

where $x=\{a=b\}$ means that the value of $x$ is equal to 1 if $a=b$ and is equal to 0 otherwise.

Test 2: The proportion of UEs positioned in the handover region to the total amount of UEs belonging to the $\mathrm{BS}$, which is expressed as

$$
\mathcal{H}_{i}=\frac{\sum_{k=1}^{\mathcal{S}_{i}} \mathcal{L}_{\mathbf{i}}}{\mathcal{S}_{i}}
$$

Where $\mathcal{H}_{i}$ refers to the handover ratio of $\mathrm{BS} i, \mathcal{S}_{i}$ is the number of UEs connected to $i$ and $\mathcal{L}_{i}$ denotes the number of UEs served by $i$ and happen to be located within the region overlapped with neighboring cells

$$
\mathcal{L}_{i}(k, j)=\left\{\begin{array}{cc}
1 & \frac{\Gamma_{i, k}}{\Gamma_{j, k}}<\beta \\
0 & \text { Otherwise }
\end{array},\right.
$$

Test 3: The total number of UEs connected to BS $i, \mathcal{S}_{i}$

$$
\mathcal{S}_{i}=\sum_{k=1}^{K}\{\zeta(k, i)=w\}
$$

Test 4: The total sum of received signal power of all UEs connected to $\mathrm{BS} i$, which is given by $\mathcal{P}_{i}$

$$
\mathcal{P}_{i}=\sum_{k=1}^{\mathcal{S}_{i}}\left[\Gamma_{i, k}\right]
$$

Test 5: The sum of interference power received by the $i$ 's user from neighboring BSs, $\mathcal{I}_{i}$

$$
\mathcal{I}_{i}=\sum_{k=1}^{\mathcal{S}_{i}} \sum_{\substack{j=1 \\ j \neq i}}^{B}\left[\Gamma_{j, k}\right]
$$

\section{Resource Partitioning}

The resource partitioning scheme is responsible for managing the inter-cell interference by orthogonalizing the resources between the conflicting BSs/users [19]. Given the interference map, $\zeta$, the maximum number of resources that BS $i$ offers each of its connected UEs, $\Omega_{i}$, is measured by dividing the available resources, $N$, by the total number of detected users.

$$
\Omega_{i}=N /\left(\sum_{k=1}^{K}\{\zeta(k, i)=1\}+\sum_{k=1}^{K}\{\zeta(k, i)=w\}\right)
$$

where the number of detected users include the UEs served by BS $i$ in addition to the interfered users that are located in the coverage range of BS $i$ which are determined based on the SIR threshold $\beta$ as discussed in sec. III. This value is measured and stored in the vector $\Omega_{B} \in \mathbb{R}^{1 \times B}$ where $\Omega_{B}=\left[\Omega_{1}, \ldots, \Omega_{i}, \ldots, \Omega_{j}, \ldots, \Omega_{B}\right]$. A given user $k$ connected to BS $i$ may not necessarily be allocated the maximum number of resources $\Omega_{i}$. For instance, if $\Omega_{j}$ of a neighboring BS $j$ is less than $\Omega_{i}$, the number of allocated resource to user $k$ is decreased to reduce the interference to the neighboring users close from BS $i$. Therefore, the notation $\Psi_{k}$ is used to denote the number of resources allocated to user $k$ which is determined as follows: (a) $\Psi_{k}$ is set to $\Omega_{i}$ if $\Omega_{j}$ is not less than $\Omega_{i}$, (b) $\Psi_{k}$ is set to $\Omega_{j}$ if $\Omega_{j}$ is less than $\Omega_{i}$ to allow BS $j$ a wider range of orthogonal resource for its own users where the vector $\Psi_{K}=\left[\Psi_{1}, \ldots, \Psi_{k} \cdots \Psi_{K}\right]$ stores the number of resources dedicated for each user.

\section{Small Cell Deactivation Algorithm}

The small cell deactivation algorithm uses the deactivation criteria and incorporates the resource partitioning scheme to switch off the unwanted BSs that when switched off, the interference reduces, the resource re-utilization improves and the capacity is increased. This objective is pursued while taking measures to prevent compromising the outage probability of the users that are connected to the switched off cells. The small cell deactivation algorithm is performed as follows

- Before beginning to execute the deactivation tests, the initial sum of PRBs allocated to all the users in the system is determined according to sec. IV-C, to be later compared with the updated value after the deactivation

$$
\Psi_{\text {tot }}=\sum_{i=1}^{B} \sum_{k=1}^{\mathcal{S}_{i}} \sum_{n=1}^{N}\left[\mathcal{A}_{k, n} \cdot \chi_{k, n}\right]
$$

The notations $\Psi_{t o t}$ and $\Psi_{\text {tot }}^{\prime}$ are used to denote the current and previous total utilized resources respectively.

- Since the NoC is one of the parameters that significantly affects the reuse efficiency as will be shown later in sec. $\mathrm{V}$, Test 1 is applied first to determine the BS with the maximum NoC

$$
\wp=\max (\mathrm{NoC})
$$




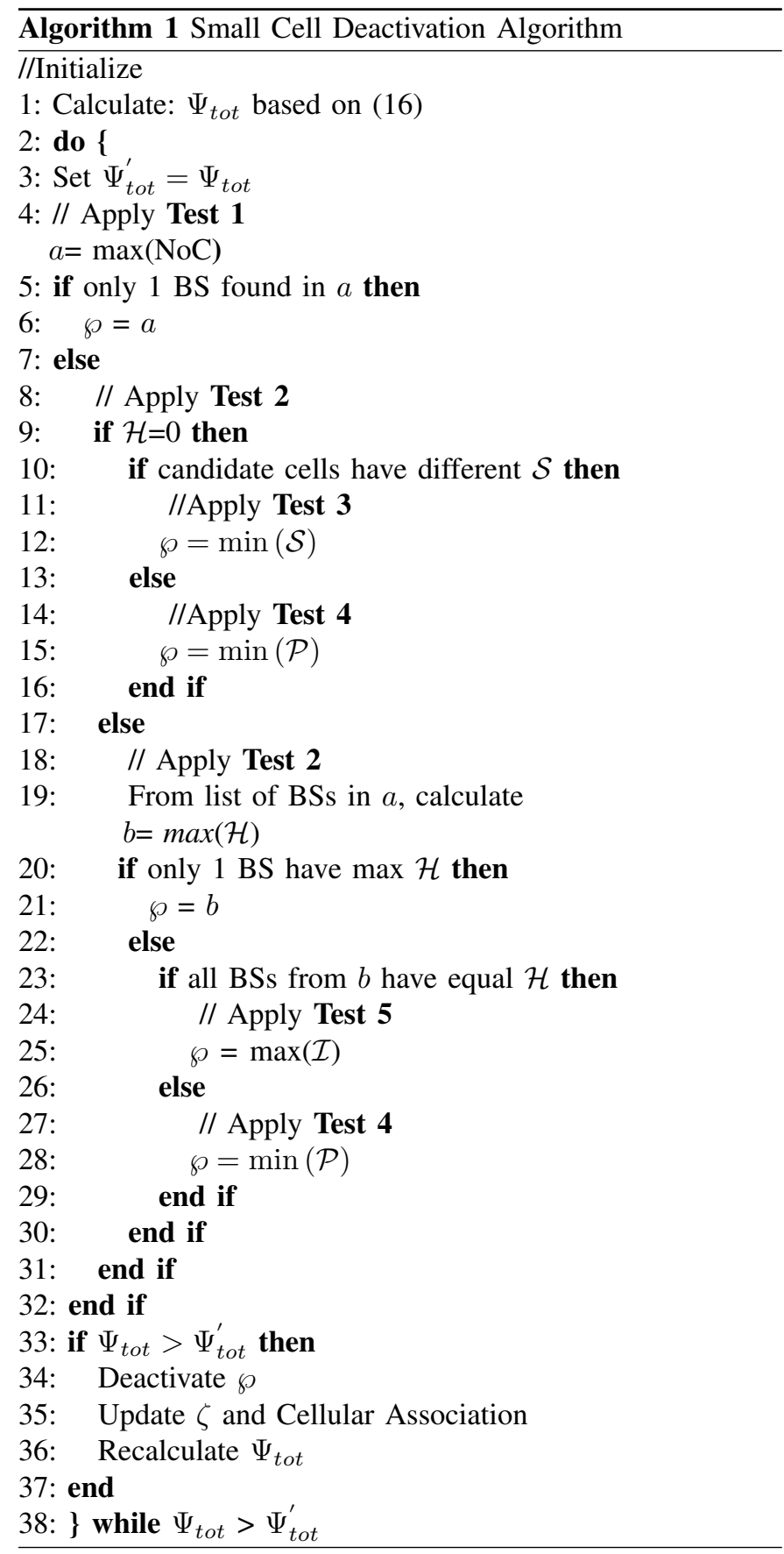

where, $\wp$ refers to the selected BS.

If only one BS is found, this BS is selected for switch off. Otherwise, in case there are multiple candidates, Test 2 is applied to identify the BS with the maximum handover ratio

$$
\wp=\max (\mathcal{H})
$$

- In case none of the UEs of the nominated BS are located in the handover region of nearby cells, the BS to be deactivated is selected based on Test 4 in case all BSs have equal number of users

$$
\wp=\min (\mathcal{P}),
$$

The reason for applying (19) is to ensure that the selected $\mathrm{BS}$ is the one that offers its users less received signal power than other candidates. This is done to enhance the overall capacity. Otherwise, Test 3 is applied and the BS with the minimum connected users is selected to reduce the probability of dropping UEs

$$
\wp=\min (\mathcal{S}),
$$

- If the nominated BSs have UEs in the handover region of other cells, the BS that has the largest handover ratio is selected to minimize the number of dropped UEs. In case more than one BS have equal handover ratio $\mathcal{H}$, and all their connected users happen to be located in the coverage area of nearby cells, the selection is performed based on Test 5 as follows

$$
\wp=\max (\mathcal{I}) .
$$

The reason for executing the test in (21) is to select the BS with the users that are receiving the maximum interference from nearby cells, since such UEs are more likely to achieve better signal quality when handed off to these cells. Otherwise, the selection decision is performed based on (19).

- Finally, the selected BS is deactivated and the disconnected users are handed off to nearby active BSs with the maximum received signal power. However, in case a user is not settled within the coverage range of any cell, this user will be dropped.

- The interference map, $\zeta$ is then updated and $\Psi_{t o t}$, is recalculated.

- The updated $\Psi_{t o t}$ is compared with the previous $\Psi_{t o t}^{\prime}$ and the selected BS $\wp$ is set to SL mode if $\Psi_{t o t}$ is greater than $\Psi_{t o t}^{\prime}$. The process then repeats to search for other BSs for deactivation.

These steps are formulated as a pseudo code structure in Algorithm1.

\section{E. Resource Allocation}

The frequency resource allocation algorithm is implemented independently by each BS after measuring the amount of available resources per user as discussed in the previous part. BSs use the CQI reports from their UEs in order to select the preferred PRBs for each user [20]. Once the preferred PRBs are determined, the allocation map, $\mathcal{A}$, that is managed by the BSs is updated.

\section{Performance Analysis}

In this section, the Probability Density Function (PDF) of the Downlink Signal-to-Interference Ratio (SIR) is derived conditionally on the user location. This PDF is used to study the effect of the SIR threshold on some key parameters in 
our system and to verify to our simulation results. While the model was simplified to a grid model with stationary BSs positions it also considers the randomness of the user location to accurately model the received interference.

\section{A. Signal-to-Interference Model}

The received signal power from $\mathrm{BS}, i$, to its served UE $k$ is given by $\mathcal{P}_{t} d_{i, k}^{-\varphi}$, where the transmission power of $i$ is represented by $\mathcal{P}_{t}$, the distance between $i$ and $k$ is denoted by $d_{i, k}$ and the pathloss exponent is referred to as $\varphi$ and the interference power received from a nearby $\mathrm{BS}, j$, can be expressed as $\mathcal{P}_{t} d_{j, k}^{-\varphi}$. If we assume $\mathcal{P}_{t}$ is identical for all BSs, user $k$ is not considered interfered by $\mathrm{BS} j$ when the recieved interference from this $\mathrm{BS}$ is lower than the threshold

$$
\mathcal{P}_{t} d_{j, k}^{-\varphi}<\frac{\mathcal{P}_{t} d_{i, k}^{-\varphi}}{\beta}
$$

Which can be simplified and rewritten as

$$
\frac{d_{i, k}}{d_{j, k}}<\left(\frac{1}{\beta}\right)^{\frac{1}{\varphi}}
$$

Where $\beta$ denotes the SIR threshold, $d_{i, k} / d_{j, k}$ is the distance ratio between $k, i$ and $j$ and is denoted by $\mathcal{D}$. Therefore, user $k$ is detected in the coverage of $\operatorname{BS} j$ if $\left(\frac{1}{\beta}\right)^{\frac{1}{\varphi}} \leq \mathcal{D} \leq 1$ and is not detected when $0<\mathcal{D}<\left(\frac{1}{\beta}\right)^{\frac{1}{\varphi}}$.

\section{B. Distance Ratio Analysis}

Consider a grid model where two BSs denoted by $P_{1}$ and $P_{2}$ are located at the center of each square. Assuming $P_{1}$ and $P_{2}$ are positioned on the $\mathrm{x}, \mathrm{y}$-plane with $P_{1}$ located at the origin, and $P_{2}$ located at a fixed distance from $P_{1}$ denoted by $\ell$, at position $\left(x_{p_{2}}, y_{p_{2}}\right)$. Assume a user $m$, is randomly and uniformly distributed in the circular area of $P_{1}$ whose location is given by $(r, \theta)$. Assuming $r$ and $\theta$ are both random variables following a uniform distribution, the pdf of $r, f_{R}(r)$, is given by

$$
f_{R}(r)=\left\{\begin{array}{cc}
\frac{1}{R} \quad, \quad 0<r \leq R \\
0 \quad, \quad \text { elsewhere }
\end{array}\right.
$$

And the pdf of $\theta, f_{\phi}(\theta)$ is given by

$$
f_{\phi}(\theta)=\left\{\begin{array}{cc}
\frac{1}{2 \pi} \quad, \quad 0 \leq \theta \leq 2 \pi \\
0 \quad, \quad \text { elsewhere }
\end{array}\right.
$$

Using trigonometric functions, $r$ and $\theta$ which represent the polar coordinates are transformed to the corresponding Cartesian coordinates $x$ and $y$ as follows

$$
\begin{aligned}
& x=r \cdot \cos (\theta) \\
& y=r \cdot \sin (\theta)
\end{aligned}
$$

The random variable $\theta$, can be transformed into the form $\cos (\theta)$ using the following

$$
f_{Z}(z)=f_{\theta}\left[g^{-1}(z)\right] \cdot\left|\frac{d g^{-1}(z)}{d z}\right|
$$

which gives

$$
f_{Z}(z)=2 \cdot\left(\frac{1}{2 \pi} \cdot\left|\frac{-1}{\sqrt{1-z^{2}}}\right|\right)
$$

where the term in (29) is multiplied by 2 for symmetry.

Therefore, $f_{Z}(z)$ can be re-written as

$$
f_{Z}(z)=\left\{\begin{array}{ccc}
\frac{1}{\pi \sqrt{1-z^{2}}} & , \quad-1<z<1 \\
0 & , \quad \text { elsewhere }
\end{array}\right.
$$

Similarly, the probability density of the transformation of $\sin (\theta)$, is given by

$$
f_{V}(v)=\left\{\begin{array}{ccc}
\frac{1}{\pi \sqrt{1-v^{2}}} \quad & -1<v<1 \\
0 & , \quad \text { elsewhere }
\end{array}\right.
$$

The probability density, $f_{X}(x)$ as stated in (26) can be expressed as

$$
f_{X}(x)=\int_{-\infty}^{+\infty} f_{Z / X}\left(\frac{z}{x}\right) \cdot f_{Z}(x) \cdot \frac{1}{x} d x
$$

where $X=Z$ and $\frac{Z}{X}=R$. From (24) and (30), $f_{X}(x)$ is given by

$$
f_{X}(x)=\frac{1}{\pi R} \cdot \int_{\frac{x}{R}}^{1} \frac{1}{x} \cdot \frac{1}{\sqrt{1-x^{2}}} d x
$$

Therefore, the pdf of $x$ can be written as

$f_{X}(x)=\left\{\begin{array}{cc}\frac{1}{\pi R} \cdot\{\log (R)-\log (x)+ & ,-R \leq x \leq R /\{0\} \\ \left.\log \left(1+\sqrt{1-\frac{x^{2}}{R^{2}}}\right)\right\} & \\ 0 & , \quad \text { elsewhere }\end{array}\right.$

The euclidean distance between $P_{2}$ and $m$ is defined by

$$
G=\sqrt{\Delta x^{2}+\Delta y^{2}}
$$

Where $\Delta x=x-x_{p_{2}}$ and $\Delta y=y-y_{p_{2}}$

And the distance ratio between $P_{1}, P_{2}$ and $m$ is given by

$$
\mathcal{D}=G / \ell
$$

Assuming $x_{p_{2}}$ and $y_{p_{2}}$ are equal to a constant value denoted by $a$, the PDF of $\Delta X, f_{\Delta X}(x)$ can be obtained using (28) and the density function of $\Delta x, f_{\Delta x}(x)$ can be written as

$f_{\Delta X}(x)=\left\{\begin{array}{cc}\frac{1}{\pi R} \cdot\{\log (R)-\log (x-a) & a-R \leq x \leq a+R /\{a\} \\ \left.+\log \left(1+\sqrt{1-\frac{(x-a)^{2}}{R^{2}}}\right)\right\} & \\ 0 & \text { elsewhere }\end{array}\right.$

In a similar fashion, the density function of $\Delta y, f_{\Delta y}(y)$ is given by 
$f_{\Delta Y}(y)=\left\{\begin{array}{cc}\frac{1}{\pi R} \cdot\{\log (R)-\log (y-a) & a-R \leq y \leq a+R /\{a\} \\ \left.+\log \left(1+\sqrt{1-\frac{(y-a)^{2}}{R^{2}}}\right)\right\} & \\ 0 & \text { elsewhere }\end{array}\right.$

The joint probability density of $\Delta x$ and $\Delta y$ can be defined as

$$
f_{\Delta X, \Delta Y}(x, y)=f_{\Delta X}(x) \cdot f_{\Delta Y}(y)
$$

The pdf of $G, f_{G}(g)$ as described in (35) can be expressed as

$$
f_{G}(g)=\int_{-\infty}^{+\infty}\left|\frac{g}{\sqrt{g^{2}-x^{2}}}\right| \cdot f_{\Delta X, \Delta Y}\left(x, \sqrt{g^{2}-x^{2}}\right)
$$

The expressions in (37) and 38 can be simplified into quadratic form as follows

$$
\begin{gathered}
f_{\Delta X}(x)=p_{1} x^{2}+p_{2} x+p_{3} \\
f_{\Delta Y}(y)=p_{1}\left(g^{2}-x^{2}\right)+p_{2} \sqrt{g^{2}-x^{2}}+p_{3}
\end{gathered}
$$

Therefore, the pdf of $G, f_{G}(g)$ can be written as

$$
f_{G}(g)=\left\{\begin{array}{ccc}
f_{G_{1}}(g) & , & \sqrt{2} l_{1}<g<\sqrt{l_{1}^{2}+l_{2}^{2}} \\
f_{G 2}(g) & , & \sqrt{l_{1}^{2}+l_{2}^{2}}<g<\sqrt{2} l_{2} \\
0 & , & \text { elsewhere }
\end{array}\right.
$$

Where $l_{1}=a-R, l_{2}=a+R$ and $f_{G_{1}}(g), f_{G_{2}}(g)$ are given by (44) and (45)

$$
\begin{gathered}
f_{G_{1}}(g)=2 g p_{2} p_{3}\left[\sqrt{g^{2}-l_{1}^{2}}-l_{1}\right]+\frac{g p_{2}^{2}}{2}\left[g^{2}-2 l_{1}^{2}\right] \\
+g p_{1} p_{3}\left[g^{2} \arctan \left(\frac{\sqrt{g^{2}-l_{1}^{2}}}{l_{1}}\right)-g^{2} \arctan \left(\frac{l_{1}}{\sqrt{g^{2}-l_{1}^{2}}}\right)\right] \\
+\frac{g p_{1}^{2}}{8}\left[2 l_{1} \sqrt{g^{2}-l_{1}^{2}}\left(g^{2}-2 l_{1}^{2}\right)+g^{4} \arctan \left(\frac{\sqrt{g^{2}-l_{1}^{2}}}{g}\right)\right. \\
\left.-g^{4} \arctan \left(\frac{l_{1}}{\sqrt{g^{2}-l_{1}^{2}}}\right)\right]
\end{gathered}
$$

$$
\begin{gathered}
f_{G_{2}}(g)=2 g p_{2} p_{3}\left[l_{2}-\sqrt{g^{2}-l_{2}^{2}}\right]+\frac{g p_{2}^{2}}{2}\left[2 l_{2}^{2}-g^{2}\right] \\
+g p_{1} p_{3}\left[g^{2} \arctan \left(\frac{l_{2}}{\sqrt{g^{2}-l_{2}^{2}}}\right)-g^{2} \arctan \left(\frac{\sqrt{g^{2}-l_{2}^{2}}}{l_{2}}\right)\right] \\
+\frac{g p_{1}^{2}}{8}\left[2 l_{2} \sqrt{g^{2}-l_{2}^{2}}\left(l_{2}^{2}-g^{2}\right)+g^{4} \arctan \left(\frac{l_{2}}{\sqrt{g^{2}-l_{2}^{2}}}\right)\right. \\
\left.-g^{4} \arctan \left(\frac{l_{1}}{\sqrt{g^{2}-l_{1}^{2}}}\right)\right]
\end{gathered}
$$

Finally, the expression in (43) can be transformed as described in equation (36) using (28) in order to obtain the pdf of the distance ratio, $f_{D}(d)$ as follows
$f_{D}(d)=\left\{\begin{array}{ccc}f_{D_{1}}(d) & , & \left(\sqrt{2} l_{1}\right) / \ell<g<\left(\sqrt{l_{1}^{2}+l_{2}^{2}}\right) / \ell \\ f_{D_{2}}(d) & , & \left(\sqrt{l_{1}^{2}+l_{2}^{2}}\right) / \ell<g<\left(\sqrt{2} l_{2}\right) / \ell \\ 0 & , & \text { elsewhere }\end{array}\right.$

Where $f_{D_{1}}(d)$ and $f_{D_{2}}(d)$ are defined as in (47) and (48)

\section{Number of Conflicts Analysis}

As the $\mathrm{NoC}$ is one of the parameters that has a major impact on the BS deactivation decision, the effect of the SIR threshold, $\beta$, on the NoC in the system is investigated based on the analysis in sec. V-A and using the PDF in (46). If we assume a grid scenario is used with $\mathcal{F}$ BSs and $\mathcal{S}_{f}$ UEs connected to BS $f$. Therefore, the number of interfered users that are detected in the coverage area of BS $f$ is given by

$$
\mathcal{L}_{1}=\mathcal{S}_{f}(\mathcal{F}-1)\left[\operatorname{Pr}\left(\left(\frac{1}{\beta}\right)^{\frac{1}{\varphi}} \leq \mathcal{D}_{i, j} \leq 1\right)\right]
$$

eq. (49) can be re-written as

$$
\mathcal{L}_{1}=\mathcal{S}_{f}(\mathcal{F}-1)\left\{1-F_{D_{i, j}}\left(\left(\frac{1}{\beta}\right)^{\frac{1}{\varphi}}\right)\right\}
$$

where $F_{D_{i, j}}\left(d_{i, j}\right)$ is the cumulative distribution function (CDF) of $f_{D_{i, j}}\left(d_{i, j}\right)$.

The number of $f$ 's users interfered by neighboring cells is given by

$$
\mathcal{L}_{2}=\mathcal{S}_{f}\left\{1-F_{D_{i, j}}\left(\left(\frac{1}{\beta}\right)^{\frac{1}{\varphi}}\right)\right\}
$$

Where $\mathrm{NoC}_{f}$ is equal to $\mathcal{L}_{1}$ added to $\mathcal{L}_{2}$. Fig. 2 illustrates the SIR threshold effect on the NoC under various distances between the cell of interest and the neighboring cells. The graph shows a gradual rise in the NoC as the SIR threshold increases. This is because increasing the threshold increases the number of detected interfered users which leads to higher NoC. It can be also noticed that the NoC is higher when the neighboring cells are closer to the cell of interest.

\section{Reuse Efficiency Analysis}

The reuse efficiency of the system is highly influenced by the SIR threshold, therefore, the effect of the SIR threshold, $\beta$, on the resource utilization of the system is analyzed in this section. The PDF in (46) is used to study the resource utilization of the system. Assuming a system with $\mathcal{F}$ BSs and $\mathcal{S}_{f}$ user per BS, the number of detected users by BS $f$ is equivalent to

$$
\mathcal{T}_{f}=\mathcal{S}_{f}+\mathcal{S}_{f}(\mathcal{F}-1)\left\{1-F_{D_{i, j}}\left(\left(\frac{1}{\beta}\right)^{\varphi}\right)\right\}
$$




$$
\begin{gathered}
f_{D_{1}}(d)=\frac{d \ell p_{1}^{2}}{8}\left[2 l_{1} \sqrt{(d \ell)^{2}-l_{1}^{2}}\left((d \ell)^{2}-2 l_{1}^{2}\right)+(d \ell)^{4} \arctan \left(\frac{\sqrt{(d \ell)^{2}-l_{1}^{2}}}{(d \ell)}\right)-(d \ell)^{4} \arctan \left(\frac{l_{1}}{\sqrt{(d \ell)^{2}-l_{1}^{2}}}\right)\right]+\frac{d \ell p_{2}^{2}}{2}\left[(d \ell)^{2}-2 l_{1}^{2}\right] \\
\quad d \ell p_{1} p_{3}\left[(d \ell)^{2} \arctan \left(\frac{\sqrt{(d \ell)^{2}-l_{1}^{2}}}{l_{1}}\right)-(d \ell)^{2} \arctan \left(\frac{l_{1}}{\sqrt{(d \ell)^{2}-l_{1}^{2}}}\right)\right]+2 d \ell p_{2} p_{3}\left[\sqrt{(d \ell)^{2}-l_{1}^{2}}-l_{1}\right] \\
f_{D_{2}}(d)=\frac{d \ell p_{1}^{2}}{8}\left[2 l_{2} \sqrt{(d \ell)^{2}-l_{2}^{2}}\left(l_{2}^{2}-(d \ell)^{2}\right)+(d \ell)^{4} \arctan \left(\frac{l_{2}}{\sqrt{(d \ell)^{2}-l_{2}^{2}}}\right)-(d \ell)^{4} \arctan \left(\frac{\sqrt{(d \ell)^{2}-l_{2}^{2}}}{l_{2}}\right)\right]+\frac{d \ell p_{2}^{2}}{2}\left[2 l_{2}^{2}-(d \ell)^{2}\right] \\
+d \ell p_{1} p_{3}\left[(d \ell)^{2} \arctan \left(\frac{l_{2}}{\sqrt{(d \ell)^{2}-l_{2}^{2}}}\right)-(d \ell)^{2} \arctan \left(\frac{\sqrt{(d \ell)^{2}-l_{2}^{2}}}{l_{2}}\right)\right]+2 d \ell p_{2} p_{3}\left[l_{2}-\sqrt{(d \ell)^{2}-l_{2}^{2}}\right]
\end{gathered}
$$

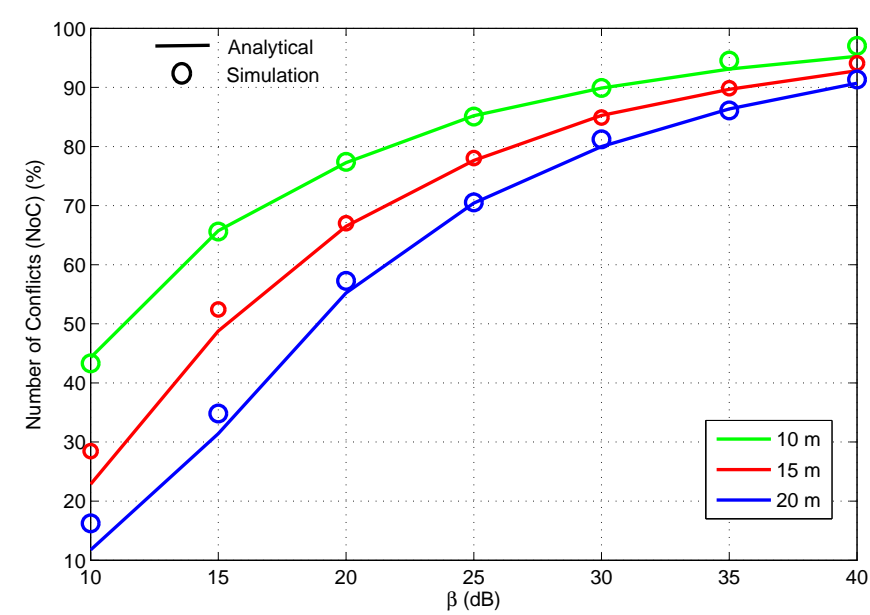

Figure 2. Impact of SIR Threshold on the NoC

The amount of available resources for each user can be expressed as $\Psi_{k}=N / \mathcal{T}_{f}$, therefore, the allocated resources per BS is given by $\Omega_{f}=\Psi_{k} \mathcal{S}_{f}$ which can be written as

$$
\Omega_{f}=\frac{N}{1+\left[(\mathcal{F}-1)\left\{1-F_{D_{i, j}}\left(\left(\frac{1}{\beta}\right)^{1 / \varphi}\right)\right\}\right]}
$$

Fig. 3 illustrates the impact of the SIR threshold on the reuse efficiency. The SIR threshold has major influence on the $\mathrm{NoC}$ which is one of the key parameters that affect the reuse efficiency of the system. It is shown that increasing the SIR threshold lowers the reuse efficiency as a result of higher NoC in the system as shown the previous section.

\section{E. Signaling Overhead}

The total amount of information exchange between the FMS and the BSs is given by

$$
d_{s}=d_{f_{i}}+d_{c}
$$

where the total number of bits required to send the interference map from BS $f_{i}$ to the FMS is denoted by $d_{f_{i}}$. Assuming BS $f_{i}$ has a total of $\mathcal{S}_{i}$ users and $J_{i}$ neighboring BSs, then $d_{f_{i}}$ is given by

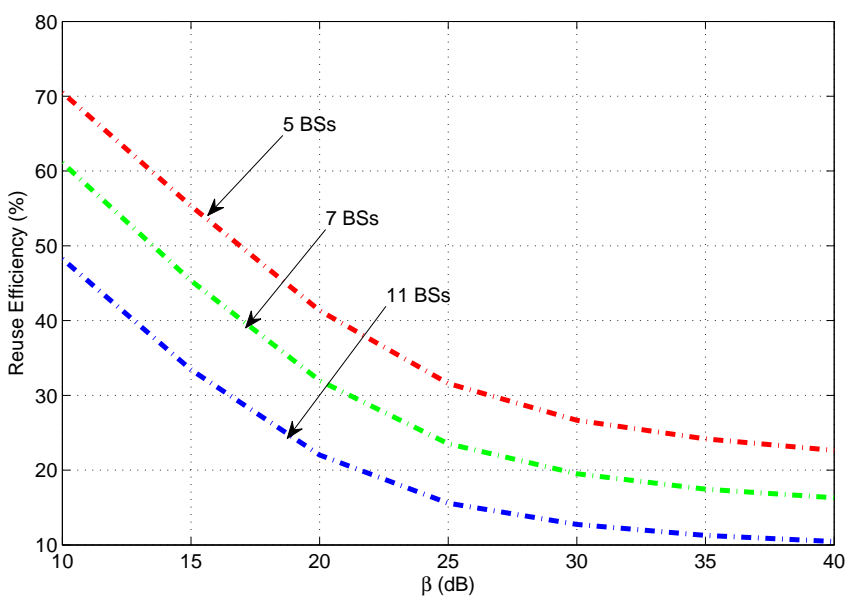

Figure 3. Impact of SIR Threshold on Resource Utilization

$$
d_{f_{i}}=\mathcal{S}_{i} \cdot d_{m} . J_{i}
$$

where, $d_{m}$ denotes the number of bits necessary to encode the local interference map of each BS. The FMS needs the local interference map feedback from each BS which can be used by the system to extract some necessary information such as the NoC, the handover ratio, $\mathcal{H}$, and the number of served users, $\mathcal{S}$. In case more than one candidate is detected, the received power of candidate BSs users needs to be fed back to the FMS

$$
d_{c}=\varrho \cdot \mathcal{S}_{i} \cdot d_{t}
$$

where $d_{t}$ denotes the number of candidate BSs for deactivation and $\varrho$ is the total needed bits to send the CQI feedback which is given by 4 bits. On the other hand, the FMS does not need to send feedback to the BSs as the allocation process is performed independently by each BS.

\section{F. Computational Complexity}

An exhaustive approach is used as an optimal benchmark in which the impact of deactivating every BS on the sum of the available resources is checked using an iterative process. In contrast, the proposed SL method is capable of selecting BSs to find the optimal outcome without the need to perform 
Table I

SIMULATION PARAMETERS

\begin{tabular}{|c|c|c|c|}
\hline Parameter & Value & Parameter & Value \\
\hline Carrier $\left(f_{c}\right)$ & $2.0 \mathrm{GHz}$ & Bandwidth & $5 / 10 \mathrm{MHz}$ \\
\hline $\begin{array}{c}\text { Resource blocks } \\
(\mathrm{RB})\end{array}$ & $25 / 50$ & $\begin{array}{c}\text { Thermal Noise } \\
\text { Density }\end{array}$ & $\begin{array}{c}-174 \\
\mathrm{dBm} / \mathrm{Hz}\end{array}$ \\
\hline $\begin{array}{c}\text { OFDM symbol } \\
\text { period }\end{array}$ & $\begin{array}{c}1.43 \times \\
10^{-4} \mathrm{~s}\end{array}$ & $\begin{array}{c}\text { Log normal } \\
\text { shadowing, } X_{\epsilon}\end{array}$ & $8 \mathrm{~dB}$ \\
\hline Wall loss, $W L$ & $18 \mathrm{~dB}$ & Fading channel & Rayleigh \\
\hline $\begin{array}{c}\text { Pathloss } \\
\text { exponent, } \varphi\end{array}$ & 3 & $\begin{array}{c}\text { Macrocell Tx } \\
\text { Power }\end{array}$ & $46 \mathrm{dBm}$ \\
\hline $\begin{array}{c}\text { Femtocell Tx } \\
\text { Power }\end{array}$ & $20 \mathrm{dBm}$ & $\begin{array}{c}\text { Macrocell } \\
\text { Radius }\end{array}$ & $289 \mathrm{~m}$ \\
\hline $\begin{array}{c}\text { Macro-Femto } \\
\text { Min Separation }\end{array}$ & $75 \mathrm{~m}$ & $\begin{array}{c}\text { Macro-UE } \\
\text { Min Separation }\end{array}$ & $35 \mathrm{~m}$ \\
\hline
\end{tabular}

an exhaustive search. To illustrate this, assume $M$ denotes the total number of BSs, which means that the loop runs for $M$ times as there are $M$ different possibilities to test. This shows that the optimal benchmark approach requires the number of iterations to be $O(M)$. On the other hand, the proposed SL method does not need iterations to search for the most appropriate small cells to be switched off.

\section{RESULTS AND PERFORMANCE ANALYSIS}

\section{A. Homogeneous Network Simulation}

A 10x10 grid model is used in this simulation to represent femtocells urban deployment where the size of each apartment is $10 \mathrm{~m} \times 10 \mathrm{~m}$ [1]. It is assumed that one femtocell and one user are randomly and uniformly dropped around the center of each apartment. Moreover, a number of femtocells are activated at random apartments following a uniform distribution. The bit rate is calculated using adaptive modulation and coding (AMC) scheme [21] and the pathloss between femtocells and UEs is given by [19]

$$
P L=P L\left(d_{0}\right)+10 \varphi \log \left(d_{i, k} / d_{0}\right)+X_{\epsilon}+W L
$$

where $d_{i, k}$ denotes the distance between BS $i$ and UE $k$, $P L\left(d_{0}\right)$ is given by $20 \log _{10}\left(4 \pi d_{0} / \lambda\right)$ where $d_{0}$ is the reference distance $(1 \mathrm{~m})$ and $\lambda$ denotes the signal wavelength. A bandwidth of $5 \mathrm{MHz}$ is utilized and the remaining simulation parameters are shown in Table 1.

The energy efficiency of the proposed SL method is measured using the energy consumption ratio (ECR) model in [22]. To evaluate the ECR of BS $i$, the consumed power by BS $i$, $P_{D L_{i}}$ is divided by the mean data rate per user, $R_{i}$

$$
E C R_{i}=P_{D L_{i}} / R_{i}
$$

The power saving of the proposed SL method is evaluated by measuring the total consumed power, $P$ [5]

$$
P=N_{\text {Active }} \cdot P_{R E}+N_{\text {Sleep }} \cdot P_{S L}
$$

Where the number of active and sleep femtocells are denoted by $N_{\text {Active }}$ and $N_{\text {Sleep }}$. The powers required to operate

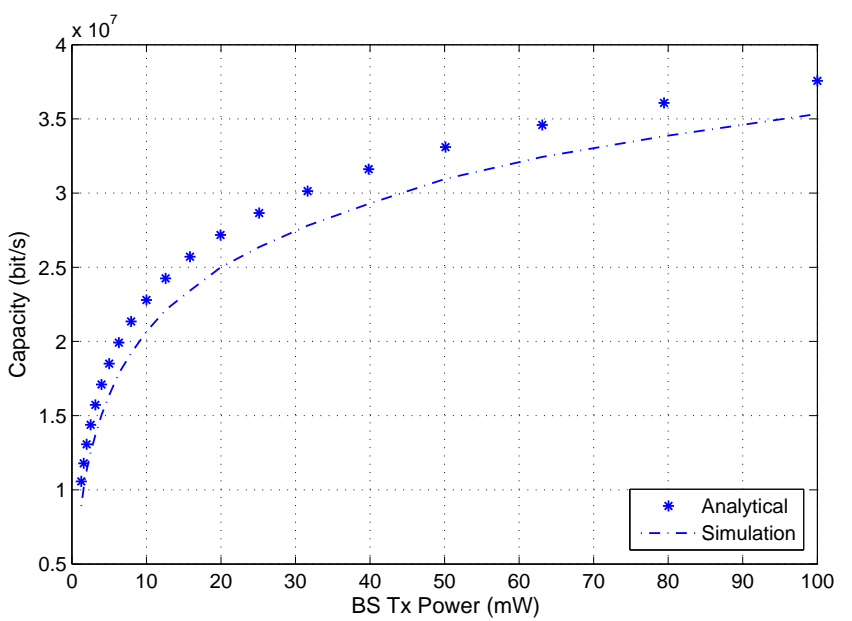

Figure 4. Upper bound for the capacity of the FSR - Always-ON system with a bandwidth of $5 \mathrm{MHz}$

the femtocell in RE and SL are represented by $P_{R E}$ and $P_{S L}$ respectively [5].

To confirm the validity of our simulation results, we have evaluated the capacity upper bound of the full spectrum reuse (FSR) - Always-ON system, which represents a generic benchmark that does not consider cell switch off. The upper bound on the capacity, $C$ can be obtained using Jensen's inequality [23]

$$
C \leq W \cdot \log _{2}\left(1+\mathbb{E}\left(\gamma_{0}\right)\right)
$$

where $\mathbb{E}\left(\gamma_{0}\right)$ is given by

$$
\mathbb{E}\left(\gamma_{0}\right)=\frac{\mathbb{E}\left(r_{0}^{-\varphi}\right)}{\eta_{0}+\sum_{b=1}^{B} \mathbb{E}\left(r_{b}^{-\varphi}\right)}
$$

Proof: See Appendix A.

The upper bound of $C$ can be obtained by substituting (61) in (60). The derived bound is numerically calculated and validated through comparison with Monte-Carlo simulation results. The upper bound for the capacity is illustrated in fig. 4 with the assumption that $\varphi$ is given by $2.7, \eta_{0}$ is 0.001 watts.

The efficiency of our proposed SL method is evaluated compared to the Manchester technique, which is based on the method in [19] in terms of using the interference map which is also applied in our method to indicate the interference between BSs and users. It uses similar assumptions as our system except that the proposed SL algorithm is not applied. Therefore, we call this method the always-ON to show the gain achieved by our proposed SL with respect to the always-ON system. Our method is also compared with an optimal approach which provides an upper bound for the system performance. Since our objective is to maximize the overall capacity, the optimal approach evaluates the effect of switching off each BS in the system on the overall throughput in each iteration and determines the BS that maximizes the overall system capacity when switched off. Normally, the first 
deactivated BS is the one that is causing the highest $\mathrm{NoC}$ in the network and is more likely to make the maximum impact when switched off. Therefore the performance of the proposed method is tested by checking the effect of switching off the first BS which is illustrated as a benchmark in the results. The threshold $\beta$ is assumed to be $20 \mathrm{~dB}$. Because SL techniques are very widely used for optimizing the power consumption in cellular systems, the method in [14] discussed in the related work will be used as a benchmark in our comparison.

The mean data rate and the ECR are illustrated in figures 5 and 6 at various densities of femtocells. It can be seen from Fig. 5 that the data rate is significantly improved in our proposed SL method in comparison to the always-ON method. This results from the improved reuse efficiency which is an outcome of reducing the interference to the nearby cells by minimizing the quantity of unnecessary active femtocells. The gap in the performance between the proposed SL method and the always-ON method is noticeably increased as the network becomes denser with an improvement of 20\%, 24.5\% and 34\% at the densities of 7,11 and 17 respectively. Fig. 6 shows that the proposed method is capable of preserving the improvement in data rate as it can be noticed that the ECR gap is increased when comparing the proposed and the always-ON methods even at higher densities. Since the objective of the benchmark method in [14] is to minimize the energy consumption, the data rate performance appears to be lower than the other methods. Fig. 7 illustrates a power consumption comparison between the proposed SL method and the other methods. Clearly, the proposed method consumes less power than the always-ON as the overall power consumption is reduced when some hardware parts such as the power amplifier are switched off during SL [5]. On the other hand, the benchmark method achieves lower power consumption compared to the other methods as it switches off more BSs. Furthermore, the performance of the benchmark FSR - Always-On method is included in fig. 5, which is seen to approach the performance of the Always-On method as the density of cells increases. This is because the FSR - Always-ON method allows BSs to utilize the entire resources where the inner users that are not exposed to interference can achieve very high data rate as opposed to the Always-ON method, which takes the inter-cell interference into account. Fig. 8 illustrates the average throughput cumulative distribution function (CDF) assuming the number of femtocells deployed in the system is 7 femtocells. It is shown that applying the proposed SL method can remarkably improve the throughout performance with approximately $30 \%$ increase compared to the always-ON method. This results from switching off the unnecessary femtocells that are causing high amount of disturbance in the surrounding environment which leads to significant reduction in the interference and allows the neighboring BSs to utilize the frequency resources more efficiently. Users are associated with BSs providing the highest received signal strength as all femtocells are assumed to use an open access policy. Fig. 9 shows a comparison between the three methods in terms of the total number of deactivated femtocells. Fig. 10 shows the ratio of dropped users vs. the total number of UEs in the system where it is shown that the ratio slightly increases at higher user densities as the proposed

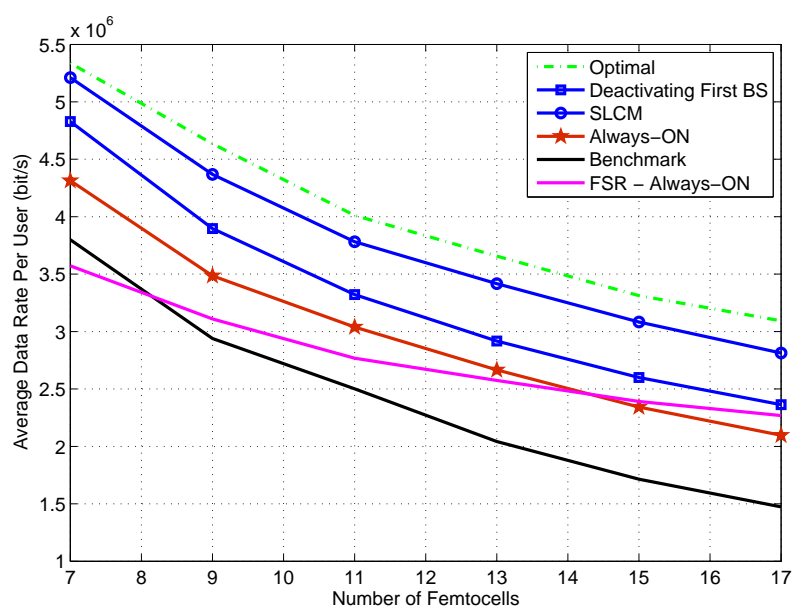

Figure 5. Data rate per user performance comparison with varying femtocell densities

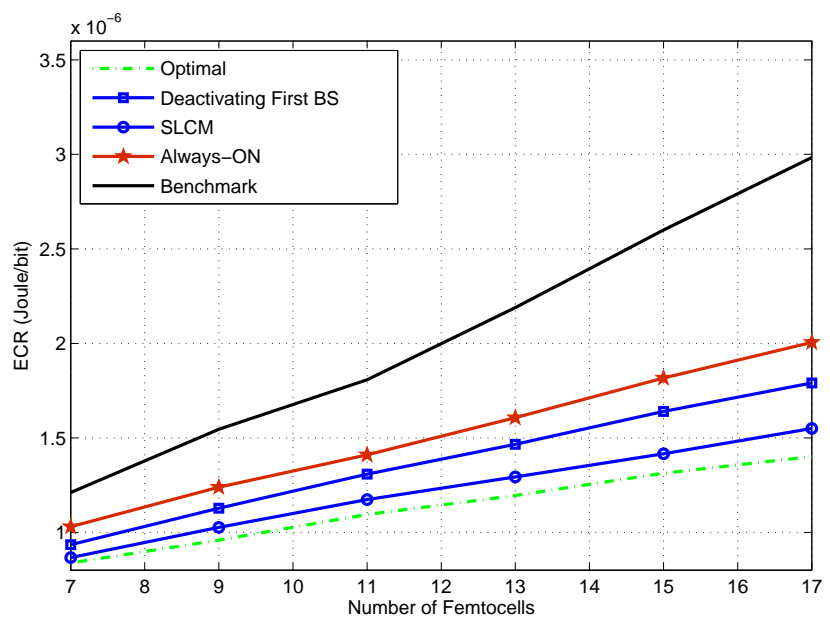

Figure 6. Energy efficiency performance comparison with varying femtocell densities

SL method aims to minimize the number of dropped UEs. Fig. 11 illustrates the average capacity per UE performance, it is clear from this figure that the proposed technique provides an average improvement between $13 \%-20 \%$ depending on the number of BSs.

\section{B. Heterogeneous Network Simulation}

In this part, we consider a suburban model with a macrocell overlaid with 10 femtocells deployed in $10 \mathrm{~m} \times 10 \mathrm{~m}$ houses which are uniformly distributed within the macrocell coverage area [1]. A total of 30 UEs are deployed with $2 / 3$ of the UEs generated in hotspots around femtocells within a radius of 10 $\mathrm{m}$ and the remaining UEs are positioned within the macrocell area in a random and a uniform manner [24]. A system bandwidth of $10 \mathrm{MHz}$ is used in this simulation with 50 PRBs available for transmission. The macrocell and femtocells are equipped with two antennas space-time-block-code (STBC) and the Stanford university interim (SUI) path loss model for Terrain type $\mathrm{C}$ is used to model the pathloss between the macrocell and all UEs [25] 


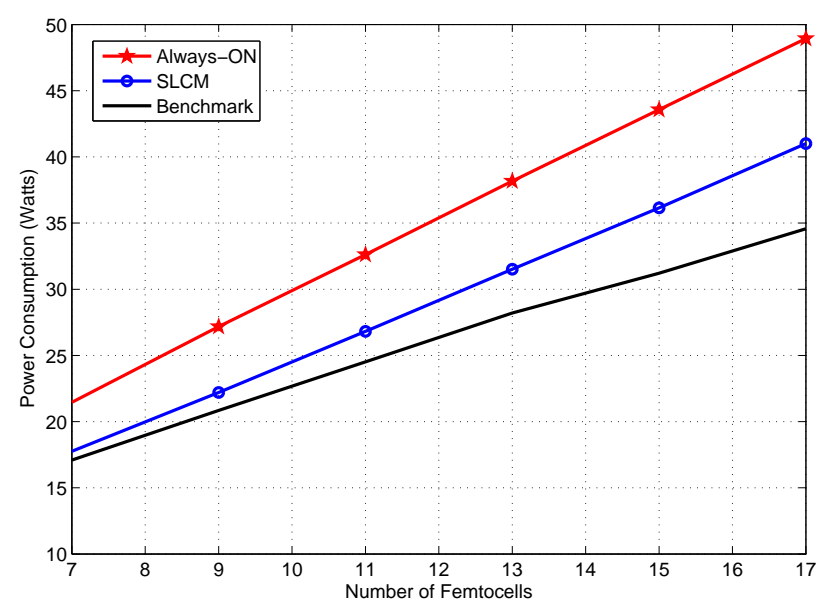

Figure 7. Power saving performance comparison with varying femtocell densities

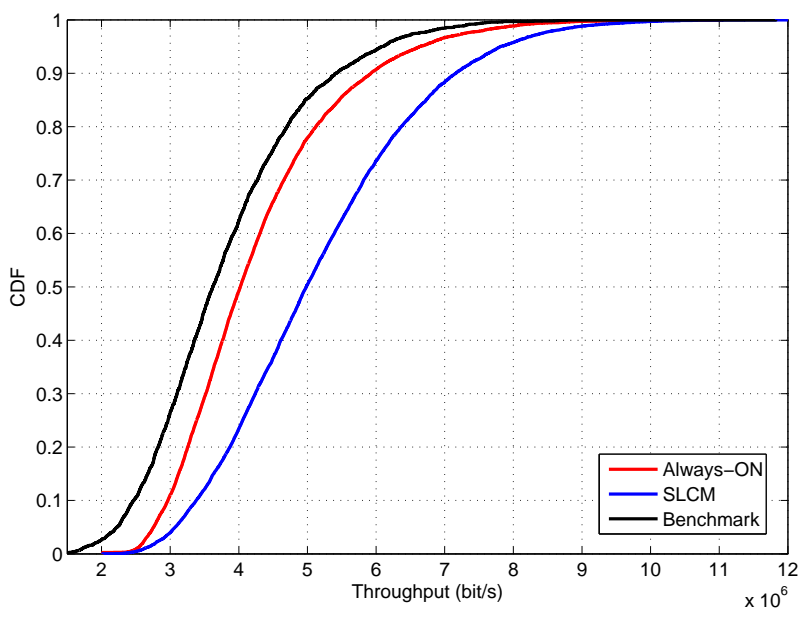

Figure 8. Femtocell network average throughput CDF with 7 deployed cells

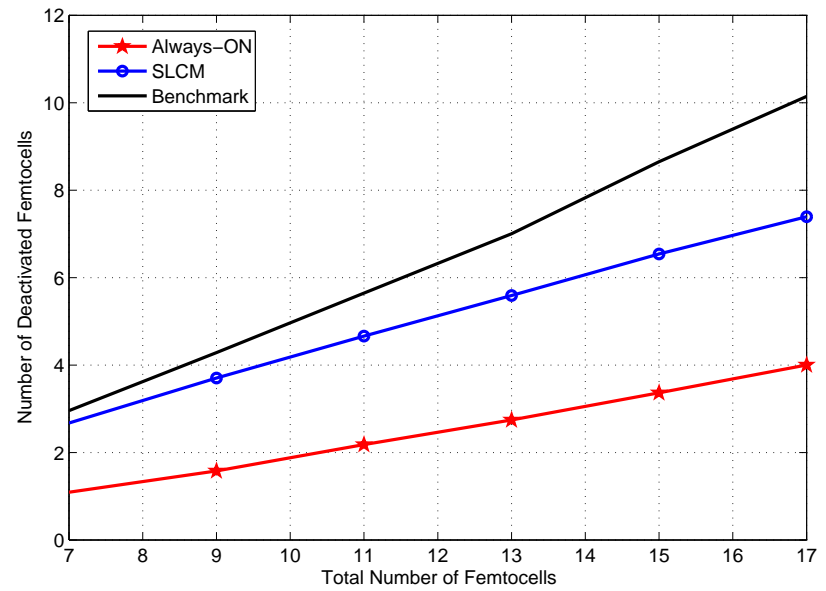

Figure 9. Number of Deactivated Femtocells Comparison

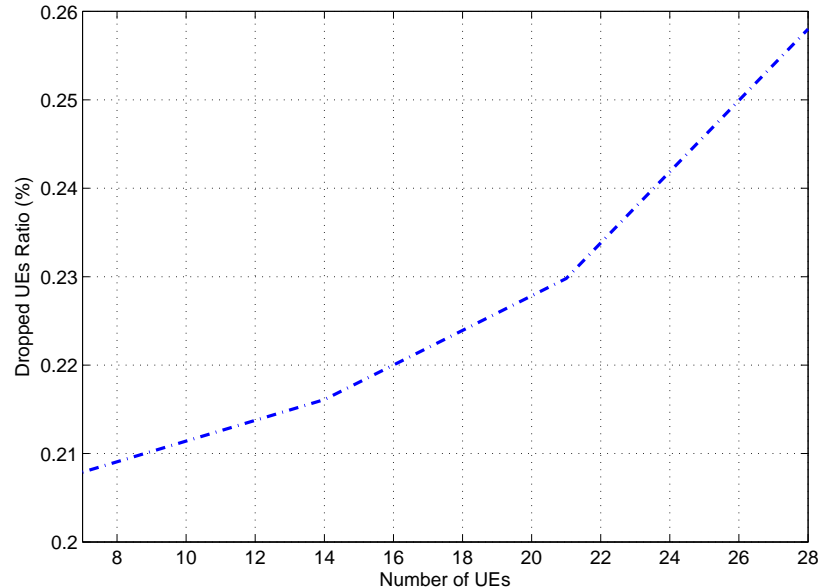

Figure 10. Dropped users ratio vs. the total number of users in the system

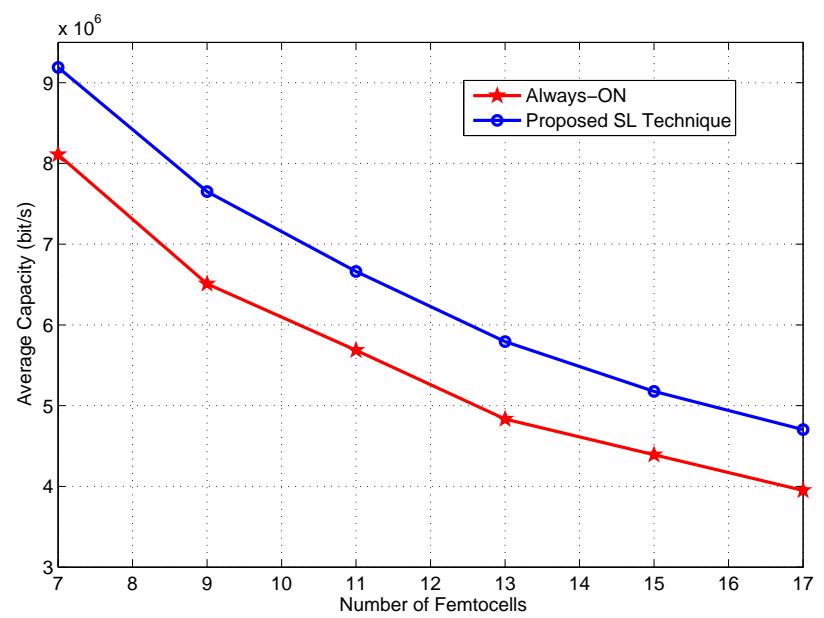

Figure 11. Average capacity performance with varying femtocell densities

$$
P L_{M}^{k}=A+10 \sigma \log \left(\frac{d_{M, k}}{100}\right)+X_{f}+X_{h}+s+W L
$$

where $A=P L\left(d_{0}=100\right), d_{M, k}$ is the distance between the macrocell and UE $k, W L$ is the wall loss, $\sigma=$ $3.6-0.005 h_{M}+\frac{20}{h_{M}}+\chi_{a} 0.59, X_{f}=6 \log \left(\frac{f_{c}}{2000}\right), X_{h}=$ $20 \log _{10}\left(\frac{2}{h_{k}}\right)$ and $s=\chi_{b}\left(8.2+\chi_{x} 1.6\right)$ where $\chi_{a}, \chi_{b}$ and $\chi_{c}$ represent Gaussian random variables. $h_{M}$ and $h_{k}$ represent the macrocell and the receiver heights which are given by $30 \mathrm{~m}$ and $2 \mathrm{~m}$ respectively.

FFR is employed to mitigate interference based on [4] where the coverage area of the macrocell is divided into inner and outer regions based on the FFR threshold which is presumed to be $20 \mathrm{~dB}$ above the noise level. The resources allocated to the inner and outer regions are $\mathcal{N} / 2$ and $\mathcal{N} / 2 \Delta$ respectively where $\Delta$ denotes the FFR reuse factor which is assumed to be 3. First, the SIRs to the macrocell and to the closest femtocell are determined by the UE which are denoted by SIR1 and SIR2 respectively, in case SIR1 is higher than the FFR threshold, the UE is considered inner and is connected to the macrocell if SIR1>SIR2 and to the femtocell otherwise. On the other hand, 


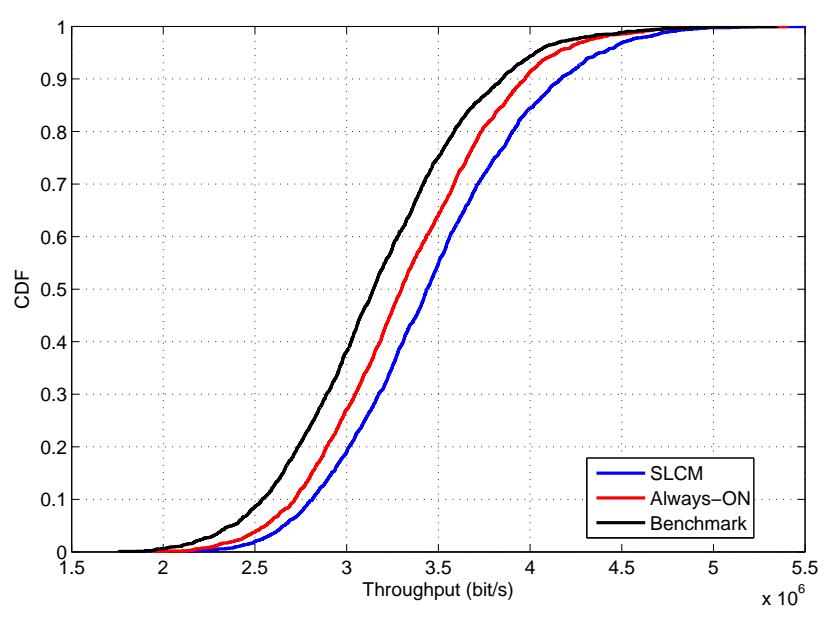

Figure 12. Heterogeneous network average throughput CDF

the UE is categorized as an outer UE if SIR1 is less than the FFR threshold. The access threshold of the femtocells, $\mu$, is adjusted to offload the macrocell UEs to the femtocells where $\mu$ is set to $12 \mathrm{~dB}$. Fig. 12 illustrates the average throughput CDF to show the performance of the proposed SL method compared to the always-ON and the benchmark methods which shows superior performance of the proposed method as compared to the other techniques. It is noticed that the performance improvement is less evident in heterogeneous network compared to the homogeneous simulation. This is due to the deployment of femtocells which are randomly positioned in the coverage area of the macrocell which in turn reduces the overlapped regions causing the number of deactivated cells to diminish.

\section{CONCLUSION}

A novel sleep mode method is presented in this paper to improve the capacity and the energy efficiency in heterogeneous networks. It was shown that performance improvements can be achieved by identifying the small cells that are positioned at undesirable interference spots and selecting them for deactivation. The results show that, compared to the always-ON system, the proposed SL method can increase the gain by up to $34 \%$ and $15.6 \%$ in terms of data rate and the energy efficiency performance, respectively. Furthermore, the CDF result showed that the throughput is improved by $30 \%$ compared with the always-on method. Finally, it was also shown that the proposed SL technique approaches the performance of the optimal benchmark with considerably lower computational complexity.

\section{APPENDIX A \\ SHANNON CAPACITY UPPER BOUND}

Consider a network with a reference $\mathrm{BS}, B_{0}$ at the origin $(0,0)$ and 6 co-channel interferers $B_{1}, \ldots B_{B}$ located at fixed distances from $B_{0}$. A reference user is located at random position $\left(r_{0}, \theta_{0}\right)$ within the area of $B_{0}$. The mean capacity of the reference user is generally expressed as

$$
C=\mathbb{E}\left[W \cdot \log _{2}\left(1+\gamma_{0}\right)\right]
$$

where the $\gamma_{0}$ is the SINR which is defined by

$$
\gamma_{0}=\frac{P_{0} \cdot \hbar \cdot r_{0}^{-\varphi}}{\eta_{0}+\sum_{b=1}^{B} P_{b} \cdot \hbar \cdot r_{b}^{-\varphi}}
$$

where, $P_{0}$ denotes the required transmission power and $P_{b}$ is the transmission power of the $b^{\text {th }}$ co-channel interferer which are assumed to be identical. $\hbar$ refers to Rayleigh fading channel which is exponentially distributed. $r_{0}$ denotes the distance between the reference BS and the reference user and $r_{b}$ is the distance between the reference user and the $b^{t h}$ cochannel interferer. The upper bound on the Shannon capacity, $C$ can be obtained using Jensen's inequality [23]

$$
C \leqslant W \cdot \log _{2}\left(1+\mathbb{E}\left(\gamma_{0}\right)\right)
$$

Evaluating the expression in (65) requires measuring the average of $\mathbb{E}\left(\gamma_{0}\right)$. From [26], the distribution of the reference user in the cell-edge region is given by

$$
f_{r_{0}}\left(r_{0}\right)=\left\{\begin{array}{ccc}
\frac{2 r_{0}}{R^{2}-R_{0}^{2}} & , \quad R_{0}<r_{0}<R \\
\frac{2 r_{0}^{2}}{R_{0}^{2}} & , \quad 0<r_{0}<R_{0}
\end{array}\right.
$$

where, $R$ denotes the cell radius and $R_{0}$ is the radius of the inner are part of the cell which are given by $10 \mathrm{~m}$ and $5 \mathrm{~m}$, respectively. Using (66), the average of $r_{0}^{-\varphi}$ can be expressed as

$$
\mathbb{E}\left(r_{0}^{-\varphi}\right)=\int_{R_{0}}^{R} r_{0}^{-\varphi} \cdot \frac{2 r_{0}}{R^{2}-R_{0}^{2}} \cdot d r_{0}
$$

that gives

$\mathbb{E}\left(r_{0}^{-\varphi}\right)=2\left(\left(-R^{2-\varphi}\right)+\left(R_{0}^{2-\varphi}\right)\right) /\left(\left(R^{2}-R_{0}^{2}\right) \cdot(\varphi-2)\right)$

The distance between the $b^{\text {th }}$ co-channel interferer and the reference user is given by [26]

$$
r_{b}=\sqrt{D^{2}+r_{0}^{2}-2 \cdot r_{0} \cdot D \cdot \cos \left(\theta_{b}+\phi_{b}\right)}
$$

where $D$ and $\phi_{b}$ represent the distance and the angle between the $B_{0}$ and the $b^{t h}$ interferer respectively, $\left(r_{b}, \theta_{b}\right)$ are the polar coordinates of the $b^{\text {th }}$ interferer related to the reference user. Based on (69), the average of $r_{b}^{-\varphi}$ can be obtained as follows

$$
\mathbb{E}\left(r_{b}^{-\varphi}\right)=\mathbb{E}\left(\left(\sqrt{D^{2}+r_{0}^{2}-2 \cdot r_{0} \cdot D \cdot \cos \left(\theta_{b}+\phi_{b}\right)}\right)^{-\varphi}\right)_{(70)}
$$

which can be written as 


$$
\begin{gathered}
\mathbb{E}\left(r_{b}^{-\varphi}\right)=\int_{R_{0}}^{R} \int_{0}^{2 \pi}\left(\sqrt{D^{2}+r_{0}^{2}-2 \cdot r_{0} \cdot D \cdot \cos \left(\theta_{b}+\phi_{b}\right)}\right)^{-\varphi} \\
f_{r_{0}}\left(r_{0}\right) \cdot f\left(\theta_{b}\right) \cdot d r_{0} \cdot d \theta_{b}
\end{gathered}
$$

The distribution of $f_{\theta_{b}}\left(\theta_{b}\right)=\frac{1}{2 \pi}$, where $0 \leq \theta_{b} \leq 2 \pi$. The expression in (71) does not have a closed-form solution and therefore the average can be obtained using numerical integration methods. The average of $\mathbb{E}\left(\gamma_{0}\right)$ can be obtained using (61).

\section{REFERENCES}

[1] X. Chu, D. Lopez-Perez, Y. Yang, and F. Gunnarsson, Heterogeneous Cellular Networks: Theory, Simulation and Deployment. Cambridge, second ed., 2008.

[2] D. Lopez-Perez, A. Valcarce, G. de la Roche, and J. Zhang, "Ofdma femtocells: A roadmap on interference avoidance," IEEE Commun. Mag., vol. 47, pp. 41-48, Sep. 2009.

[3] A. Hamza, S. Khalifa, H. Hamza, and K. Elsayed, "A survey on inter-cell interference coordination techniques in ofdma-based cellular networks," IEEE Commun. Surveys Tutorials, vol. 15, no. 4, pp. 1642-1670, 2013.

[4] T. D. Novlan, R. K. Ganti, A. Ghosh, and J. G. Andrews, "Analytical evaluation of fractional frequency reuse for heterogeneous cellular networks," IEEE Trans. Commun., vol. 60, pp. 2029-2039, July 2012.

[5] I. Ashraf, F. Boccardi, and L. Ho, "Sleep mode techniques for small cell deployments," IEEE Commun. Mag., vol. 49, pp. 72-79, Aug 2011.

[6] Y. Li, H. Celebi, M. Daneshmand, C. Wang, and W. Zhao, "Energyefficient femtocell networks: challenges and opportunities," IEEE Trans. Wireless Commun., vol. 20, pp. 99-105, December 2013.

[7] C. Jia and T. J. Lim, "Resource partitioning and user association with sleep-mode base stations in heterogeneous cellular networks," IEEE Trans. Wireless Commun., vol. 14, pp. 3780-3793, July 2015.

[8] P. Ren and M. Tao, "A decentralized sleep mechanism in heterogeneous cellular networks with qos constraints," IEEE Wireless Commun. Lett., vol. 3, no. 5, pp. 509-512, 2014.

[9] C.-Y. Chang, W. Liao, H.-Y. Hsieh, and D. shan Shiu, "On optimal cell activation for coverage preservation in green cellular networks," IEEE Trans. Mobile Comput., vol. 13, pp. 2580-2591, Nov 2014.

[10] A. Ebrahim and E. Alsusa, "Interference minimization through sleep mode based resource allocation for future femtocell networks," in Proc. IEEE Int. Conf. Commun. (ICC), pp. 1679-1684, June 2015.

[11] M. Wang, X. Zhu, Z. Zeng, S. Wan, and W. Li, "System performance analysis of ofdma-based femtocell networks," in Commun. Technology and Applicat. (ICCTA 2011), IET Int. Conf., pp. 405-410.

[12] Y. Li, Y. Jia, Y. Wang, and Q. Liu, "Collaborative sleeping scheme for femtocell networks," in Proc. IEEE Green Comput. and Commun. (GreenCom), pp. 142-147, Aug 2013.

[13] A. De Domenico, E. Strinati, and A. Duda, "An energy efficient cell selection scheme for open access femtocell networks," in Proc. IEEE Pers. Indoor and Mobile Radio Commun. (PIMRC), pp. 436-441, Sept. 2012.

[14] W. Vereecken, M. Deruyck, D. Colle, W. Joseph, M. Pickavet, L. Martens, and P. Demeester, "Evaluation of the potential for energy saving in macrocell and femtocell networks using a heuristic introducing sleep modes in base stations," EURASIP J. Wireless Commun. and Networking, vol. 2012, p. 170, 2012.

[15] S. Ali, M. Ismail, and R. Nordin, "Femtocell sleep mode activation based interference mitigation in two-tier networks," in 4th Int. Conf. on Elect. Eng. and Informatics, ICEEI 2013, pp. 2202-2207, Sept 2013.

[16] S.-F. Chou, H.-L. Chao, and C.-L. Liu, "An efficient measurement report mechanism for long term evolution networks," in Proc. IEEE Pers. Indoor and Mobile Radio Commun. (PIMRC), pp. 197-201, Sept 2011.

[17] S. Saunders and et al., Femtocells: Opportunities and Challenges for Business and Technology. Wiley, 2009.

[18] M. Ruggiero and J. Boccuzzi, Femtocells Design and Application. Mc Graw Hill, 2011.

[19] W. Pramudito and E. Alsusa, "A hybrid resource management technique for energy and qos optimization in fractional frequency reuse based cellular networks," IEEE Trans. Commun., vol. 61, pp. 4948-4960, Dec 2013.
[20] N. Kolehmainen, J. Puttonen, P. Kela, T. Ristaniemi, T. Henttonen, and M. Moisio, "Channel quality indication reporting schemes for utran long term evolution downlink," in Proc. IEEE Veh. Technol. Conf., pp. 25222526, May 2008.

[21] 3GPP, "Technical Specification Group Radio Access Network; Physical layer aspects of UTRA High Speed Downlink Packet Access," TR 25.848, 3rd Generation Partnership Project (3GPP), Mar. 2001.

[22] O. Arnold, F. Richter, G. Fettweis, and O. Blume, "Power consumption modeling of different base station types in heterogeneous cellular networks," in Future Network and Mobile Summit, 2010, pp. 1-8, June 2010.

[23] A. Goldsmith, WIRELESS COMMUNICATIONS. Cambridge University Pressl, 2005.

[24] 3GPP, "Evolved Universal Terrestrial Radio Access (E-UTRA); Further Advancements for E-UTRA Physical Layer Aspects," TS 36.814, 3rd Generation Partnership Project (3GPP), Mar. 2010.

[25] S. S. Jeng, J. M. Chen, C. W. Tsung, and Y. F. Lu, "Coverage probability analysis of ieee 802.16 system with smart antenna system over stanford university interim fading channels," IET Commun., vol. 4, no. 1, pp. 91$101,2010$.

[26] A. Mahmud and K. A. Hamdi, "A unified framework for the analysis of fractional frequency reuse techniques," IEEE Trans. on Commun., vol. 62, pp. 3692-3705, Oct 2014 